\title{
Band gap modification of single-walled carbon nanotube and boron nitride nanotube under a transverse electric field
}

\author{
Chun-Wei Chen ${ }^{1}$, Ming-Hsien Lee ${ }^{2}$ and S J Clark ${ }^{3}$ \\ ${ }^{1}$ Department of Materials Science and Engineering, National Taiwan University, Taipei, \\ Taiwan \\ ${ }^{2}$ Department of Physics, Tamkang University, Tamsui, Taipei, Taiwan \\ ${ }^{3}$ Department of Physics, University of Durham, Durham DH1 3LE, UK
}

Received 28 July 2004, in final form 13 October 2004

Published 18 November 2004

Online at stacks.iop.org/Nano/15/1837

doi:10.1088/0957-4484/15/12/025

\begin{abstract}
The electronic structures of carbon (C) and boron nitride (BN) nanotubes under a transverse electric field were investigated through the first-principles pseudopotential density-functional theory (DFT) calculations. It was found that band gap modifications occur both in the semiconducting $\mathrm{C}$ and $\mathrm{BN}$ nanotubes under an external electric field by inducing a semiconductor-metal transition. The variations of the band gap sizes with transverse electric fields are very different between $\mathrm{C}$ and $\mathrm{BN}$ nanotubes. In the semiconducting $\mathrm{C}$ nanotube, a sharp semiconductor-metal transition does not occur until a threshold electric field is achieved; the BN nanotube, on the other hand, shows a gradual reduction of the band gap size once an external electric field is applied due to the larger ionicity of BN bonds. In addition, the semiconductor-metal transition in both $\mathrm{C}$ and $\mathrm{BN}$ nanotubes occurs at a lower value of electric field with increasing diameter. The ability to tune the band gap in both $\mathrm{C}$ and $\mathrm{BN}$ nanotubes by an external electric field provides the possibility for future electronic and electro-optic nanodevice applications.
\end{abstract}

(Some figures in this article are in colour only in the electronic version)

\section{Introduction}

From the time of their discovery [1], carbon (C) and boronnitride (BN) nanotubes (CNTs and BN-NTs) have received an increasing amount interest due to their novel properties and potential applications in nanodevices. CNTs can demonstrate either semiconducting or metallic conduction depending sensitively on the tube diameter and chirality, suggesting a variety of nanoelectronics applications [2,3]. Recent calculations show that radial deformation can induce important modifications in the electronic structures and conduction properties of CNTs and BN-NTs $[4,5]$. Another way to modify the band gap of the nanotube is to apply an external electric field. The carbon nanotube field-effect transistor (CNTFET) is a promising candidate for future electronic devices [6]. The response of the nanotube to an external electric field is of interest for studying its future application, such as in logic gates, static memory cells and sensor devices. The band structure of the CNT can be modified if the transverse electric field strength is large enough to couple the neighbouring subbands, resulting in a significant change in their electronic and transport properties. The conventional one-dimensional band structure, which assumes uniform charge and potential distributions along the nanotube circumference, is therefore perturbed, leading to the modification of its band structure. Previous tight-binding calculations have indicated that CNTs may undergo band gap opening/closing under a transverse electric field depending on its helicity [7]. Such a large locally transverse electric field can be achieved in the nanotube electronic devices due to their small diameters. BN-NTs are also interesting materials due to their wide band gap values $(\sim 3.5-5.5 \mathrm{eV})$, and exhibit semiconducting properties independent of chirality and diameter. The ability to modify the band gap by an external electric field make BN-NTs a 
(a)

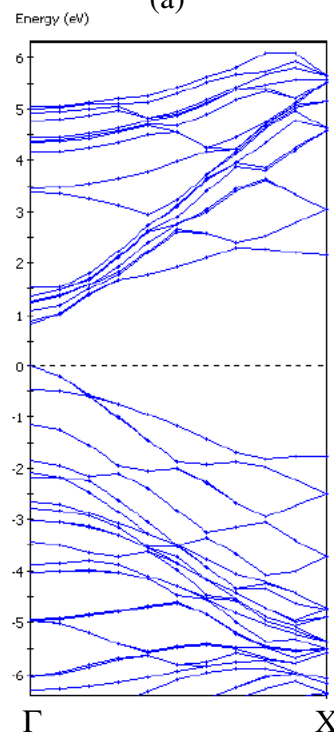

(d)

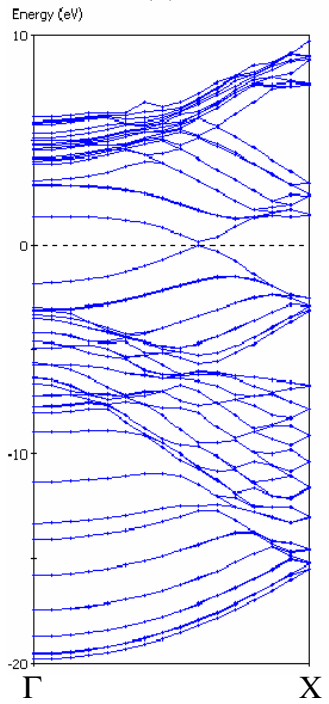

(b)

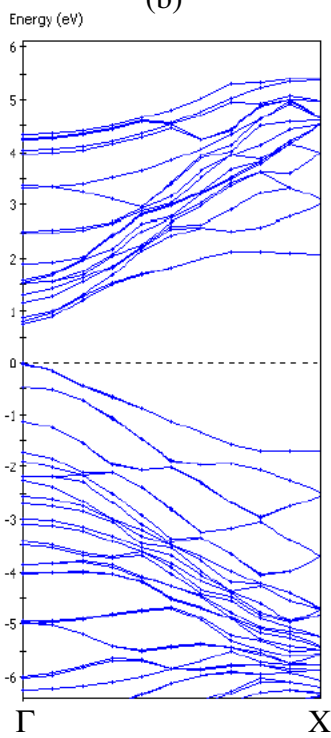

(e)

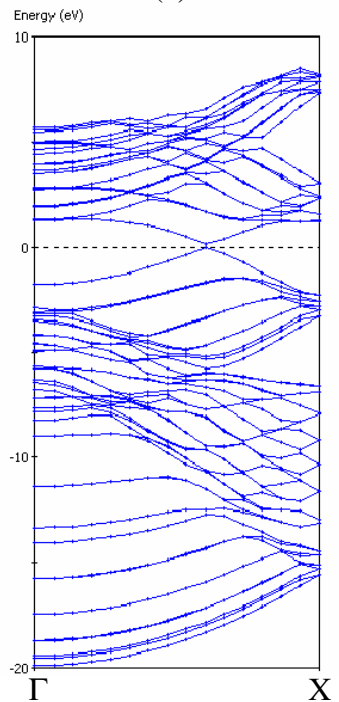

(c)

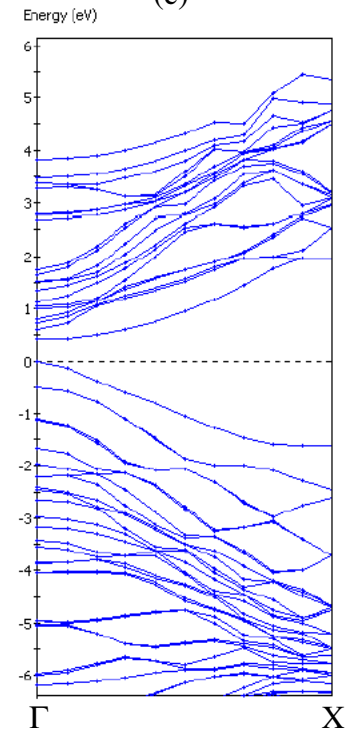

(f)

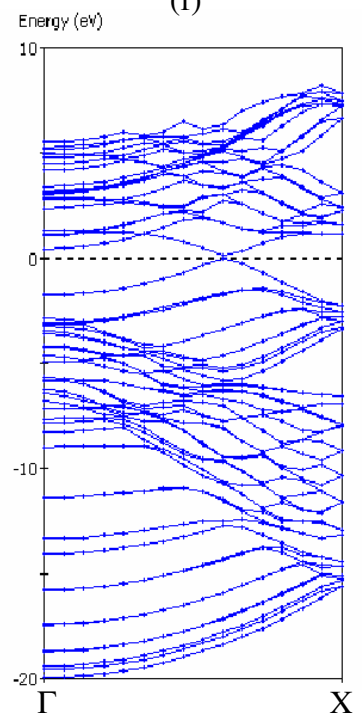

Figure 1. Band structures of (10,0) CNTs under an external electric field of (a) $0 \mathrm{~V} \AA^{-1}$, (b) $0.25 \mathrm{~V} \AA^{-1}$, (c) $0.38 \mathrm{~V} \AA^{-1}$, respectively; band structures of $(5,5)$ CNTs under an external electric field of (a) $0 \mathrm{~V} \AA^{-1}$, (b) $0.25 \mathrm{~V} \AA^{-1}$, (c) $0.38 \mathrm{~V} \AA^{-1}$, respectively.

potential candidate for electric-optical (EO) modulation device applications.

The object of this paper is to provide comparative studies of CNTs and BN-NTs under the effect of the transverse electric field based on first-principles ( $a b$ initio) total energy and electronic structure calculations. Systematic and comprehensive analyses were carried out to study the variations of the electronic properties of CNTs and BN-NTs such as band gap modification and charge redistribution under external transverse electric fields.

\section{Computational methods}

The first-principles total energy and electronic structure calculations were performed using the CASTEP code $[8,9]$, which is a plane-wave, pseudopotential program based on the density functional theory (DFT). Generalized gradient approximation (GGA) [10] was used with the exchangecorrelation potential by Wang and Perdew (PW91) [11]. The ion-electron interaction was modelled by the non-local real space [12], ultrasoft pseudopotential [13]. These simulated models were constructed within a tetragonal supercell with the lattice constants of $a, b$ and $c$. The lattice constant $b$ and $c$ were equal to $25 \AA$ to avoid the interaction between two adjacent nanotubes. The lattice constant $a$ along the tube axis was taken to be equal to the one dimensional (1D) lattice parameter of the nanotube. The tube was taken along the $x$ direction and the circular cross section was lying in the $(y, z)$ plane. Both the CNTs and BN-NTs structures were fully optimized until the force on each atom during relaxation was less than $0.005 \mathrm{eV}^{-1}$. The summation over the $1 \mathrm{D}$ Brillouin zone (BZ) with wavevectors varying only along the tube axis was carried out with a $k$-point sampling using a MonkhorstPack grid [14] with a $0.02 \AA^{-1} k$-point spacing. A kineticenergy cutoff of $280 \mathrm{eV}$ and 12 special $k$ points were used to 
Band gap modification of single-walled CNT and BN-NT under a transverse electric field

ensure the convergence in the calculations. To study the effect of the transverse electric field on the electronic structure of the nanotube, the potential generated by the external electric filed along the $z$ direction (perpendicular to the tube axis) is modelled as a sawtooth-like potential, i.e.

$$
V_{\mathrm{ext}}(r)=|e| E z\left(-\frac{L}{2}<z \leqslant \frac{L}{2}\right)
$$

where $E$ is the magnitude of the screened electric field and $L$ is the size of the supercell, chosen to be large enough so that the tube is located at the centre of the supercell to avoid discontinuity at the supercell boundary. A screened electric field is used in equation (1), since the first-principles density functional calculations [15] demonstrated that the charge screening of the external electric field can be well described simply by changing the slope of the external potential.

\section{Results and discussion}

In this study, the electronic properties of zigzag $(10,0)$ and armchair $(5,5)$ CNTs and BN-NTs were investigated, respectively. Figure 1 shows the calculated band structures of the zigzag $(10,0)$ semiconducting CNT under various external electric fields. The original unperturbed band structure of the $(10,0)$ nanotube gives a band gap of $0.8 \mathrm{eV}$ at the $\Gamma$ point, showing a similar result to the previous calculations [16]. Under an external electric field below $0.25 \mathrm{~V}^{-1}$, a slight reduction in the band gap with a value of $0.73 \mathrm{eV}$ has been found and the crossed or almost degenerated states are split in both the valence and conduction bands due to the perturbation by an external electric field, which reduces the symmetry of the wavefunction in the CNT. The subband mixing and the splitting of energy levels become more pronounced as the field strength increases as shown in figure 1. The band edge states in both the valence and conduction bands are found to move close to each other and eventually cross over each other. The onset of a semiconductor-metal transition followed by the band gap closure occurs as the electric field reaches a value above $0.30 \mathrm{~V} \AA^{-1}$ as shown in figure 2. Beyond this applied field, the band gap vanishes and the carbon nanotube becomes metallic. It is shown that below the threshold electric field $\left(0.38 \mathrm{~V} \AA^{-1}\right)$, only slight variations of the eigenvalues of the states at the $\Gamma$ point of the Brillouin zone are observed. When the electric field strength increases up to $0.38 \mathrm{~V}^{-1}$, the lowest conduction $\pi^{*}$ state shows a relatively higher rate of downward shift as shown in figure 1 (c) and the semiconductor-metal transition occurs at this stage. Once we further increase the field strength up to $0.5 \mathrm{~V} \AA^{-1}$, the above lowest conduction $\pi^{*}$ state merges with the highest valence band state at the $\Gamma$ point (not shown here), the completely metallic behaviour with the band closure for the zigzag $(10,0)$ carbon nanotube can be seen. The sharp semiconductor-metal transition in CNTs under an external transverse electric field demonstrates the typical characteristics of an electronic switch device. In figure 2, we compare the variations of the band gap sizes as a function of the field strengths for both $(10,0)$ and $(20,0)$ semiconducting CNTs with diameters 0.789 and $1.568 \mathrm{~nm}$. The electronic structure of the $(20,0) \mathrm{CNT}$ is more sensitive to the external field perturbations due to the energy separations between neighbouring subbands being smaller and the subband

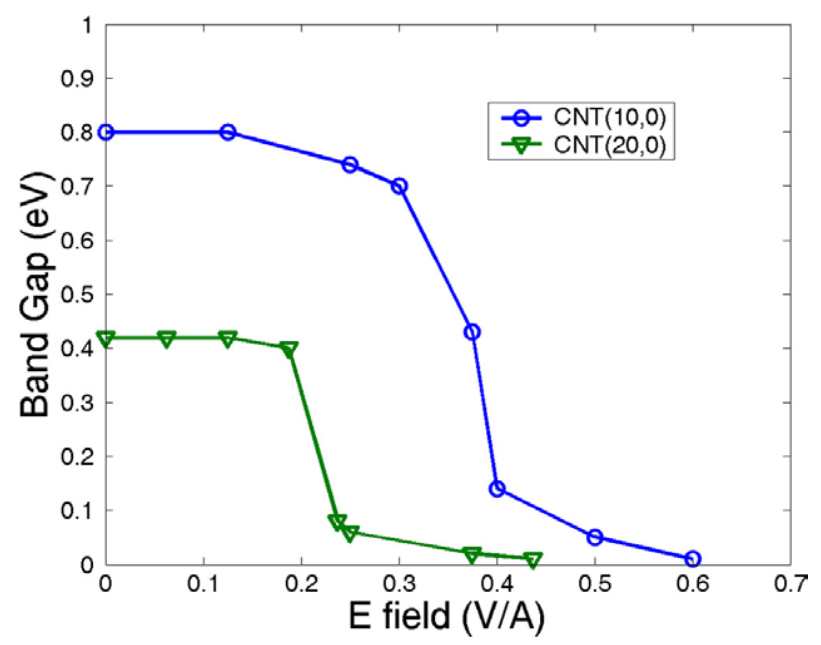

Figure 2. The band gaps of $(10,0)$ and $(20,0)$ CNTs as a function of field strength.

mixing becomes more pronounced. The $(20,0) \mathrm{CNT}$ shows a semiconductor-metal transition at an external electric field of $0.20 \mathrm{~V} \AA^{-1}$ compared to the $0.30 \mathrm{~V}^{-1}$ for the $(10,0)$ CNT. The result indicates that the threshold electric field for the semiconductor-metal transition in CNTs decreases with increasing diameter. For the CNT with a larger diameter (such as several nanometres), only a reasonably lower electric field (e.g. $<0.05 \mathrm{~V}^{-1}$ ) is required for the semiconductor-metal transition, which gives the possibility for the future application of the nanotube device acting as a quantum switch.

The variations of the band structure of the $(5,5)$ metallic CNT modified by different external electric fields are shown in figures $1(\mathrm{~d})-(\mathrm{f})$. The $(5,5) \mathrm{CNT}$ remains metallic with an enhancement of density of states around the Fermi level under an external electric field. The degenerate states at the electric field $E=0 \mathrm{~V} \AA^{-1}$ split and the splitting becomes more obvious with the increasing field strengths. However, the degree of modification of the band structure under the same electric field is less significant for the $(5,5)$ CNT than for the $(10,0) \mathrm{CNT}$. This is mainly due to the stronger $\mathrm{p}_{z}$ orbital interactions between neighbouring $\mathrm{C}$ atoms in the $(5,5) \mathrm{CNT}$ than in the $(10,0) \mathrm{CNT}$ along the field direction, leading to the $(5,5)$ CNT being more resistant to transverse electric field perturbation. A detailed discussion will be presented in the latter section.

Next, we examine the modification of the band structures of the BN-NTs under external transverse electric fields. Since the larger ionicity of the BN bonds in the hexagonal structure [17] leads to the opening of a band gap $(\sim 5.8 \mathrm{eV})$ compared to the semi-metallic graphite, the corresponding $\mathrm{BN}-\mathrm{NT}$ s exhibit semiconducting properties independent of the wrapping index. Previous tight-binding calculations show that as the tube diameter decreases, the band gap size of the zigzag tube decreases more rapidly, as compared to that of the armchair tube [17]. The band structures of the zigzag $(10,0)$ and armchair $(5,5) \mathrm{BN}-\mathrm{NT}$ s under various transverse external electric fields are compared as shown in figure 3 . The unperturbed $(10,0) \mathrm{BN}-\mathrm{NT}$ is a direct gap semiconductor with a band gap $4.05 \mathrm{eV}$, whereas the $(5,5) \mathrm{BN}-\mathrm{NT}$ is an indirect gap material with a band gap $4.20 \mathrm{eV}$ similar to the previous calculations [18]. It is expected that these band gap values are 
(a)

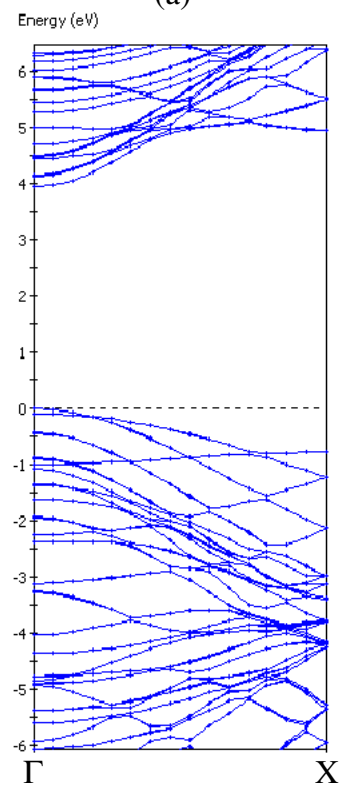

(d)

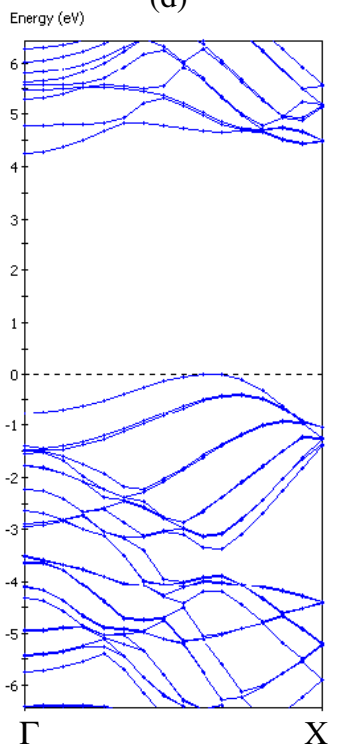

(b)

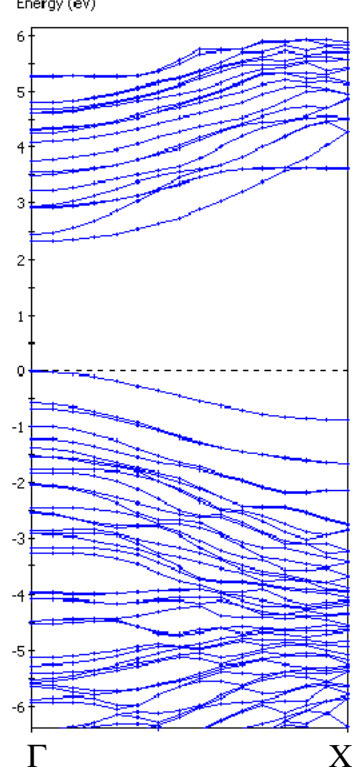

(e)

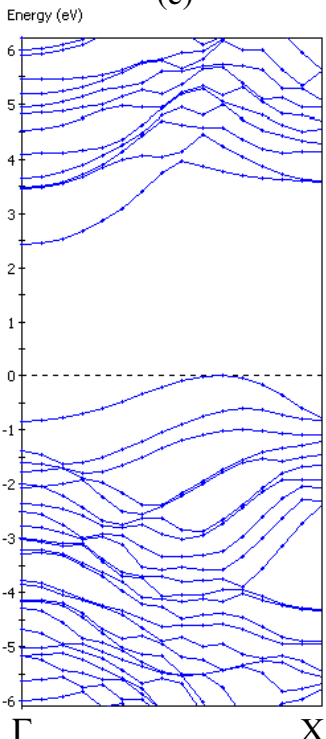

(c)

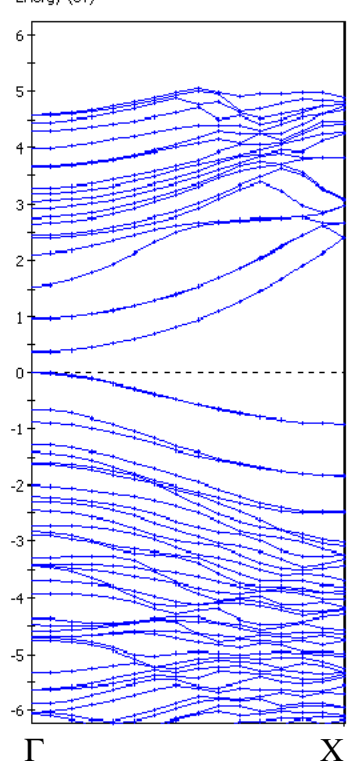

(f)

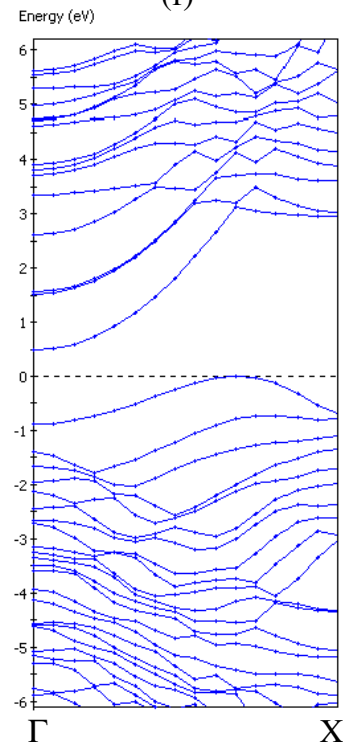

Figure 3. Band structures of $(10,0) \mathrm{BN}-\mathrm{NT}$ s under an external electric field of (a) $0 \mathrm{~V} \AA^{-1}$, (b) $0.13 \mathrm{~V} \AA^{-1}$, (c) $0.25 \mathrm{~V} \AA^{-1}$, respectively; band structures of $(5,5) \mathrm{BN}-\mathrm{NTs}$ under an external electric field of (a) $0 \mathrm{~V} \AA^{-1}$, (b) $0.13 \mathrm{~V} \AA^{-1}$, (c) $0.25 \mathrm{~V} \AA^{-1}$, respectively.

underestimated due to the ab intio LDA calculations. Band overlap in the $(5,5) \mathrm{CNT}$ occurs at a point along the $\Gamma$ to $x$ direction; for the $(5,5) \mathrm{BN}-\mathrm{NT}$, due to the anti-symmetry of the ionic potential, an opening of the band gap at this point is expected, giving an indirect band gap for the $(5,5) \mathrm{BN}-\mathrm{NT}$. As the external field strengths are enhanced, the modification of the band structures becomes more pronounced in both $(10,0)$ and $(5,5)$ BN-NTs. The band gap sizes decrease rapidly with the increasing electric fields in both cases. Both the $(10,0)$ and $(5,5) \mathrm{BN}-\mathrm{NT}$ s show a more sensitive semiconductor-metal transition behaviour under transverse electric fields compared to the CNT counterparts as shown in figure 1, with a lower threshold electric field. Figure 4 shows the evolution of the band gap sizes for zigzag BN-NTs with different diameters under various external electric fields. It is clearly shown that the semiconductor-metal transition occurs at a lower value of threshold electric field with increasing diameter. Unlike in the semiconducting CNTs where the variations of band gap values are quite small below the threshold electric field, the semiconducting BN-NTs, on the other hand, demonstrate a gradual reduction in the band gap values once the external electric field is applied. The high sensitivity to tuning the energy gap by an external electric field makes the BN-NT a promising band gap engineering material for electro-optic (EO) modulation device applications.

Next, we consider the charge redistribution of NTs under an external electric field. Figure 5 shows the smallest building block for constructing the zigzag $(10,0)$ and armchair $(5,5)$ CNT lattices. For the zigzag $(10,0)$ and armchair $(5,5)$ CNTs, respectively, the building block is the same but only with a $90^{\circ}$ 
Band gap modification of single-walled CNT and BN-NT under a transverse electric field

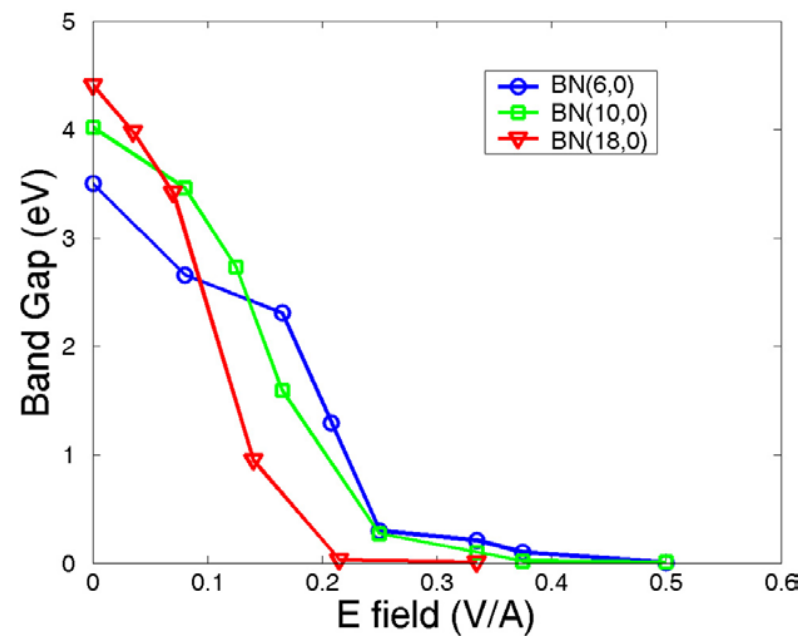

Figure 4. The band gaps of $(6,0),(10,0)$ and $(18,0)$ BN-NTs are plotted as a function of field strength.

rotation. When the electric field transverse to the tube axis is applied, it is found that the neighbouring $\mathrm{C}$ atoms in the zigzag $(10,0) \mathrm{CNT}$ are not located along the field direction; for the armchair $(5,5)$, on the other hand, the neighbouring $\mathrm{C}$ atoms are located along the field direction. Such a structural discrepancy leads to the different charge redistribution in response to an external electric field. Figure 5(a) exhibits the contour plots of the charge densities for the valence band maximum (VBM, $\pi$ state) and conduction band minimum $\left(\mathrm{CBM}, \pi^{*}\right.$ state) states in the $(10,0) \mathrm{CNT}$ with and without external electric fields. For the unperturbed $(10,0) \mathrm{CNT}$, the charge densities are found to be distributed evenly along the circumferential direction both in the VBM and CBM states.
Under the transverse electric field $\left(0.3 \mathrm{~V} \AA^{-1}\right)$, the symmetric distributions of charge densities break, giving the VBM states on the upper part and the CBM states on the lower part of the $(10,0) \mathrm{CNT}$. The CNT is now polarized with an induced dipole moment created by an external electric field in terms of a higher electron charge density in the lower part than that in the upper part. For the armchair $(5,5) \mathrm{CNT}$, the asymmetric charge distribution as shown in figure 5 (b) is not so pronounced as found in the $(10,0) \mathrm{CNT}$ under the same external electric field. This is mainly due to the stronger $\mathrm{p}_{z}$ orbital interactions of neighbouring $\mathrm{C}$ atoms along the field direction in the armchair CNT, giving that the $(5,5) \mathrm{CNT}$ is more resistant to the external electric field perturbation compared to the $(10,0) \mathrm{CNT}$. This is also consistent with the above result that the modification of the band structure of the $(5,5) \mathrm{CNT}$ in figure 1 is less sensitive to the transverse electric field perturbation.

For the unperturbed $(10,0)$ and $(5,5) \mathrm{BN}-\mathrm{NTs}$, it is found that the $\operatorname{VBM}\left(\pi\right.$ state) mostly consists of $\mathrm{p}_{z}$ orbitals around $\mathrm{N}$ atoms, while the CBM ( $\pi^{*}$ state) is composed of $\mathrm{p}_{z}$ orbitals distributed around the $\mathrm{B}$ atoms due to the higher electron affinity of the $\mathrm{N}$ atoms. The result indicates that the $\mathrm{p}_{z}$ orbitals are located only at one type of atom for these states. BN-NTs show a higher degree of charge localization compared to the CNTs counterparts. When the transverse electric field is applied, the redistributed charges are more localized in the certain atoms both in $(10,0)$ and $(5,5)$ BN-NTs. For the VBM state, charges are mostly located at the $\mathrm{N}$ atoms in the upper part of the BN-NT; for the CBM state, they are mostly localized at the $\mathrm{B}$ atoms in the lower part as shown in figure 6 . The degree of the charge redistribution is more sensitive to an external electric field for the BN-NTs than for the CNTs due to the higher ionicity of the BN bond. This gives a lower threshold electric field for the semiconductor-metal transition in BN-NTs. (a)

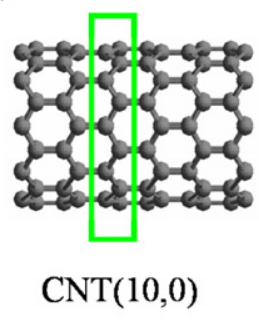

(b)

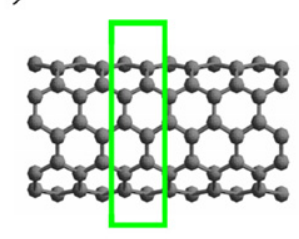

$\operatorname{CNT}(5,5)$
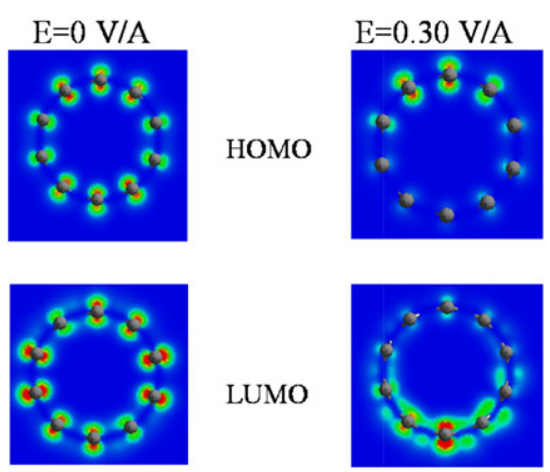

E field
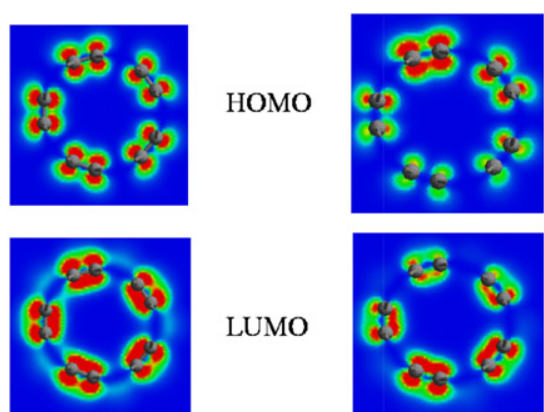

LUMO

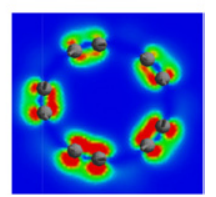

Figure 5. Charge redistribution in the VBM and CBM states for (a) $(10,0)$ and (b) $(5,5)$ CNTs under an external electric field of 0 and $0.30 \mathrm{~V} \AA^{-1}$, respectively. 
(a)

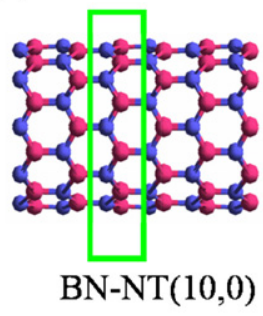

(b)

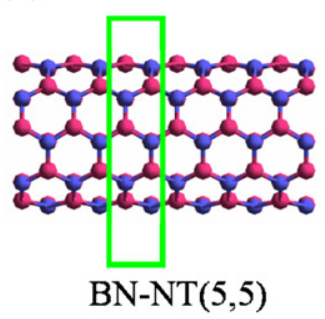

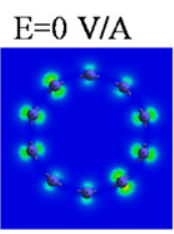
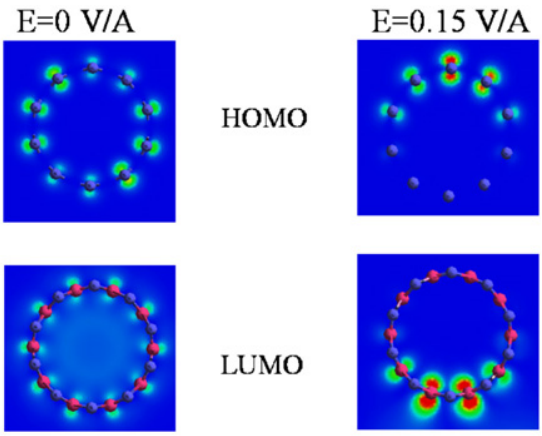

E field

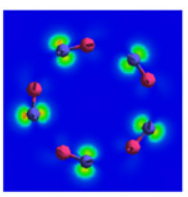

HOMO
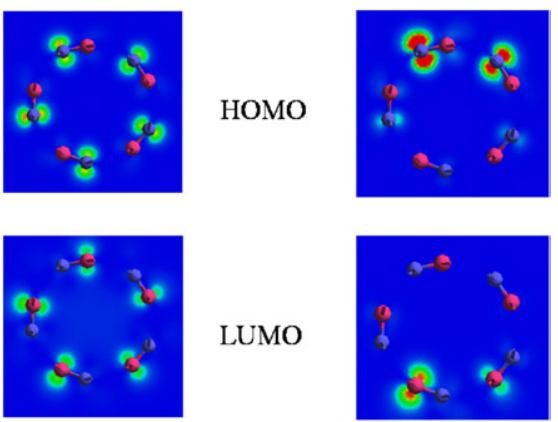

Figure 6. Charge redistribution in the VBM and CBM states for (a) $(10,0)$ and (b) $(5,5)$ BN-NTs under an external electric field of 0 and $0.15 \mathrm{~V} \AA^{-1}$, respectively.

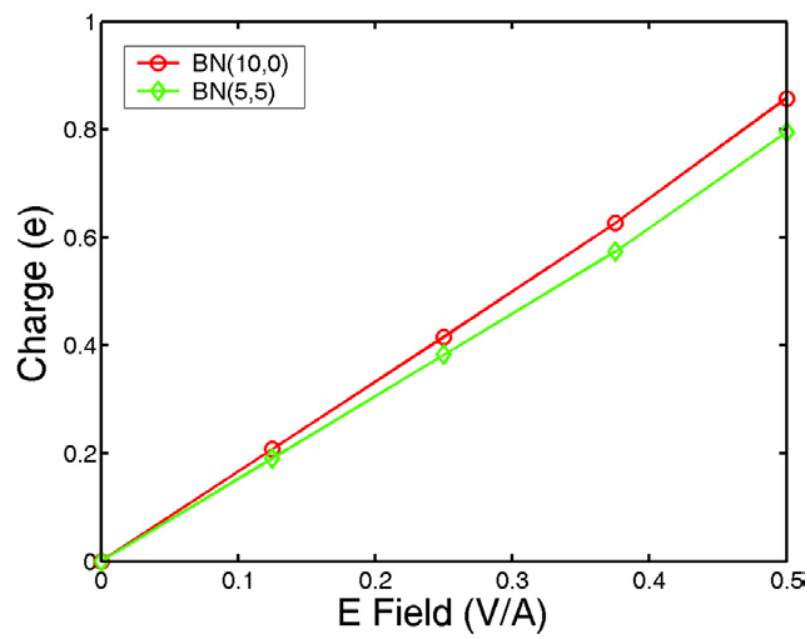

Figure 7. Evolution of the charge redistribution for the upper half $\mathrm{BN}$ nanotubes as a function of the electric fields for the $(10,0)$ and $(5,5)$ BN-NTs.

In addition, the zigzag $(10,0) \mathrm{BN}-\mathrm{NT}$ exhibits a larger degree of charge redistribution than the armchair $(5,5) \mathrm{BN}-\mathrm{NT}$. This is mainly due to the larger distance between same species along the circumferential in the $(5,5) \mathrm{BN}-\mathrm{NT}$ than in the $(10,0)$ BN-NT, which makes it less sensitive to an external electric field. Figure 7 shows the evolution of the charge redistribution which is defined as the total charge difference for the upper half $\mathrm{BN}$ nanotubes as a function of the external electric fields for the $(10,0)$ and $(5,5) \mathrm{BN}-\mathrm{NT}$, respectively. This result indicates that the zigzag $(10,0) \mathrm{BN}-\mathrm{NT}$ will be more sensitive to the modification of the band gap under an external transverse electric field than the armchair $(5,5)$ BN-NT.

\section{Conclusion}

In this work, we have performed extensive first-principles pseudopotential density-functional theory calculations on Cand BN-NTs under transverse electric fields. We have found the ability for band gap engineering in both semiconducting C- and BN-NTs by applying an external transverse electric field. The variations of the band gap sizes with transverse electric fields are very different from those in both $\mathrm{C}$ and $\mathrm{BN}$ nanotubes. It was found that the variations of band gap values in semiconducting CNTs are quite small below the threshold electric field; the BN-NTs, on the other hand, show a gradual reduction of band gap values once an external electric field is applied due to larger ionicity of $\mathrm{BN}$ bonds. In addition, it was found that the threshold electric field for semiconductor-metal transition in both C- and BN-NTs decreases with increasing diameter. The above results provide the possibility of band gap tuning by an external transverse electric field, which may be useful for future electronic and electro-optic nanodevice applications.

\section{Acknowledgments}

This work is supported by National Science Council, Taiwan (Project No. NSC93-2120-M-002-010) and the US Airforce project (Project No. AFOSR-AOARD-04-4023).

\section{References}

[1] Iijima S 1991 Nature 56345

[2] Reed M A and Tour J M 2000 Sci. Am. 28286

[3] Tan S J, Verschueren A R M and Dekker C 1998 Nature 393 49

[4] Gulseren O, Yildirim T, Ciraci S and Kilic C 2002 Phys. Rev. B 65155410

[5] Kim Y-H, Chang K J and Louie S G 2001 Phys. Rev. B 63 205408 
Band gap modification of single-walled CNT and BN-NT under a transverse electric field

[6] Appenzeller J, Knoch J, Derycke V, Martel R, Wind S and Avouris Ph 2002 Phys. Rev. Lett. 89126801

[7] Kim Y-H and Chang K J 2001 Phys. Rev. B 64153404 Li Y, Rotkin S V and Ravaioli U 2003 Nano Lett. 3183

[8] Pyane M C, Teter M, Allan D C and Joannopoulos J D 1992 Rev. Mod. Phys. 641045

[9] Mailman V, Winkler B, White J A, Pickard C J, Payne M C, Akhmatskaya E V and Nobes R H 2000 Int. J. Quantum Chem. 77 895-910

[10] Perdew J P, Chevary J A, Vosko S H, Jackson K A, Pederson M R, Singh D J and Fiolhais C 1992 Phys. Rev. B 46 6671-87
[11] Wang Y and Perdew J P 1991 Phys. Rev. B 438911 Wang Y and Perdew J P 1991 Phys. Rev. B 4413298

[12] King-Smith R D, Pyane M C and Lin J S 1991 Phys. Rev. B 4413063

[13] Vanderbilt D 1990 Phys. Rev. B 41 7892-5

[14] Monkhorst H J and Pack J D 1976 Phys. Rev. B 135188

[15] Kunc K and Resta R 1982 Phys. Rev. Lett. 48406

[16] Reich S, Thomsen C and Ordejón P 2002 Phys. Rev. B 65 155411

[17] Zunger A, Katzir A and Halperin A 1974 Phys. Rev. B 135660

[18] Rubio A, Corkill J L and Cohen M L 1994 Phys. Rev. 495081 